\title{
Single nucleotide replacement in the Atlantic salmon genome using CRISPR/Cas9 and asymmetrical oligonucleotide donors
}

Anne Hege Straume ${ }^{1}$, Erik Kjærner-Semb ${ }^{1}$ Kai Ove Skaftnesmo ${ }^{1}$, Hilal Güralp ${ }^{1}$, Simon Lillico ${ }^{2}$, Anna Wargelius ${ }^{1}$ and Rolf Brudvik Edvardsen ${ }^{1 *}$

\begin{abstract}
Background: New breeding technologies (NBT) using CRISPR/Cas9-induced homology directed repair (HDR) has the potential to expedite genetic improvement in aquaculture. The long generation time in Atlantic salmon makes breeding an unattractive solution to obtain homozygous mutants and improving the rates of perfect HDR in founder (FO) fish is thus required. Genome editing can represent small DNA changes down to single nucleotide replacements (SNR). This enables edits such as premature stop codons or single amino acid changes and may be used to obtain fish with traits favorable to aquaculture, e.g. disease resistance. A method for SNR has not yet been demonstrated in salmon.

Results: Using CRISPR/Cas9 and asymmetrical ODNs, we were able to perform precise SNR and introduce a premature stop codon in dnd in F0 salmon. Deep sequencing demonstrated up to 59.2\% efficiency in single embryos. In addition, using the same asymmetrical ODN design, we inserted a FLAG element into slc45a2 and dnd, showing high individual perfect HDR efficiencies (up to 36.7 and $32.7 \%$, respectively).

Conclusions: In this work, we demonstrate that precise SNR and knock-in (KI) can be performed in F0 salmon embryos using asymmetrical oligonucleotide (ODN) donors. We suggest that HDR-induced SNR can be applied as a powerful NBT, allowing efficient introgression of favorable alleles and bypassing challenges associated with traditional selective breeding.
\end{abstract}

Keywords: HDR, ODN, Gene editing, Knock-in, Aquaculture, New breeding technologies

\section{Background}

There is an increasing demand for sustainable animal husbandry, and the fast-growing fish aquaculture industry is a food production sector with great potential to improve global food security. Fish aquaculture is also considered to be efficient in terms of feed conversion and protein retention compared to most terrestrial livestock [1, 2]. Atlantic salmon (Salmo salar L.) is farmed

\footnotetext{
* Correspondence: rolfbe@hi.no

${ }^{1}$ Institute of Marine Research, P.O. Box 1870, Nordnes, NO-5817 Bergen, Norway

Full list of author information is available at the end of the article
}

in the sea at a large scale, but further growth is currently hindered by a range of issues including genetic introgression of escapees into wild populations and the spread of disease [3, 4]. New breeding technologies (NBT) using gene editing offer an exciting opportunity to increase the sustainability of open sea-cage salmon farming by allowing us to induce both sterility and disease resistance $[2,5-7]$.

A CRISPR/Cas9 induced double-stranded DNA break (DSB) in the coding sequence of a gene, followed by activation of the endogenous non-homologous end joining (NHEJ) pathway, results in an array of unpredictable

C C The Author(s). 2021 Open Access This article is licensed under a Creative Commons Attribution 4.0 International License, which permits use, sharing, adaptation, distribution and reproduction in any medium or format, as long as you give appropriate credit to the original author(s) and the source, provide a link to the Creative Commons licence, and indicate if changes were made. The images or other third party material in this article are included in the article's Creative Commons licence, unless indicated otherwise in a credit line to the material. If material is not included in the article's Creative Commons licence and your intended use is not permitted by statutory regulation or exceeds the permitted use, you will need to obtain permission directly from the copyright holder. To view a copy of this licence, visit http://creativecommons.org/licenses/by/4.0/ The Creative Commons Public Domain Dedication waiver (http://creativecommons.org/publicdomain/zero/1.0/) applies to the data made available in this article, unless otherwise stated in a credit line to the data. 
insertions or deletions that may result in frameshift and gene knock-out (KO). This is a useful approach to study KO phenotypes, and has been applied successfully in salmon $[5,7,8]$ and several other farmed fish species [924]. To make precise genome alterations, it is a necessity to induce homology directed repair (HDR) by supplying a repair template homologous to the CRISPR target site, thereby allowing to change SNPs, insert affinity tags for protein detection and modify regulatory elements to alter expression of target genes. An example is channel catfish, where HDR mediated editing was used to increase disease resistance [25]. A single nucleotide replacement (SNR) can be used to introduce favorable wild type alleles and could be a promising and time saving solution compared to traditional breeding with backcrossing and selection. The genetic progress in selective breeding programs is also limited by the heritability of the target traits, and the standing genetic variation in the broodstock. NBT using CRISPR/Cas9-induced HDR can offer new solutions and opportunities in these areas $[2,26]$. An important issue when it comes to gene editing in salmon, is to reduce mosaicism in individual founder (F0) fish. The long generation time (3-4 years) makes breeding an unattractive option to obtain homozygous mutants, and most functional studies must be performed in F0. Thus, improving the efficiency of perfect editing in F0 individuals is crucial.

We have previously demonstrated highly efficient HDR in salmon using symmetrical oligonucleotides (ODNs) with short $(24 / 48 / 84 \mathrm{bp})$ homology arms to knock-in (KI) a FLAG element in the pigmentation gene solute carrier family 45 member 2 (slc45a2). Using highthroughput sequencing (HTS), we showed in vivo ODNmediated $\mathrm{KI}$ in almost all the gene edited animals and demonstrated perfect HDR integration rates of up to $27 \%$ in individual F0 embryos [27]. Short homology arms have also been shown to induce efficient HDR in zebrafish $[28,29]$.

In this work, we aimed to perform SNR and increase the rates of perfect HDR in individual F0 salmon. Asymmetrical ODNs in combination with CRISPR/Cas9 have previously been demonstrated to improve HDR rates in cell cultures [30] and induced pluripotent stem cells [31]. Using asymmetrical ODNs, we have successfully performed SNR and introduced a premature stop codon in the primordial germ cell survival factor gene dead end $(d n d)$. In addition, also using asymmetrical ODNs, we have inserted FLAG elements into both slc45a2 and dnd. SNR was more efficient than FLAG KI, suggesting that HDR efficiency may be inversely proportional with insert size. As previously [27], we found HDR efficiency to be dependent on template concentration, but suggest using the lowest possible concentration to avoid toxicity and enable targeting multiple genes at the same time.
Our results show that CRISPR/Cas9 in combination with asymmetrical ODNs enables rapid and precise changes to the genome in individual F0 animals and present a promising tool for fish breeders in the future.

\section{Results \\ FLAG KI targeting slc45a2 and dnd}

Targeting slc45a2 [5] and dnd [7], we have here performed KI of a FLAG element in F0 salmon using CRIS PR/Cas9 and asymmetrical ODNs (Fig. 1a and Fig. S1). Analyzing the rate of perfect HDR in individual animals by HTS of amplicons, we detected an average of $13.6 \%$ (std 10.9\%) for slc45a2 and 7.6\% (std 10.1\%) for dnd (Fig. 1b). Interestingly, individuals in both groups displayed a very high efficiency with up to $36.7 \%$ perfect HDR in slc45a2, and $32.7 \%$ in dnd (Table S1). This is higher than our previously reported results, using symmetrical ODNs at $1.5 \mu \mathrm{M}$ [27]. When comparing the efficiency of FLAG KI (targeting slc45a2) using asymmetrical ODNs described herein, to symmetrical ODNs described before [27], a significant difference $(P<$ 0.0001 ) was detected for average perfect HDR between symmetrical (5.1\%) and asymmetrical ODNs (13.6\%). No significant difference was detected when comparing the average rates of erroneous HDR between symmetrical (3.1\%) and asymmetrical ODNs (2.0\%) (Fig. S2).

\section{Oligonucleotide concentration affects HDR efficiency}

We and others [27, 32] have shown that increasing the concentration of the DNA donor improves HDR efficiency. However, DNA can be toxic to cells and we wanted to elucidate if there is a trade-off between high integration efficiency and toxicity by testing the slc $45 a 2$ FLAG KI ODN at three different concentrations: 0.5, 1.5 and $4.0 \mu \mathrm{M}$ (Fig. 2). In accordance with our previous results, a template concentration of $1.5 \mu \mathrm{M}$ resulted in the most efficient KI. We detected the approximately same average efficiency when using 0.5 and $4 \mu \mathrm{M}$. However, the highest concentration resulted in fewer pure albinos and a higher degree of mosaicism compared to individuals injected with lower concentrations of template (Fig. S3). As expected, the HTS results from the animals who had received the highest dose revealed a much higher percentage of wild type reads (Table S1).

\section{Asymmetrical ODNs induce highly efficient single nucleotide replacement}

Targeting $d n d$, we performed a SNR using an asymmetrical ODN while at the same time continuing to refine the ODN concentration. Using $0.15,1.5$ and $4 \mu \mathrm{M}$ ODN concentrations, we obtained an average perfect HDR of $7.4 \%$ (std 14.8), 12.5\% (std 14.3) and 7.4\% (std 9.4), respectively (Fig. 3). However, when analyzing individual fish, the most striking result was obtained using $1.5 \mu \mathrm{M}$, 


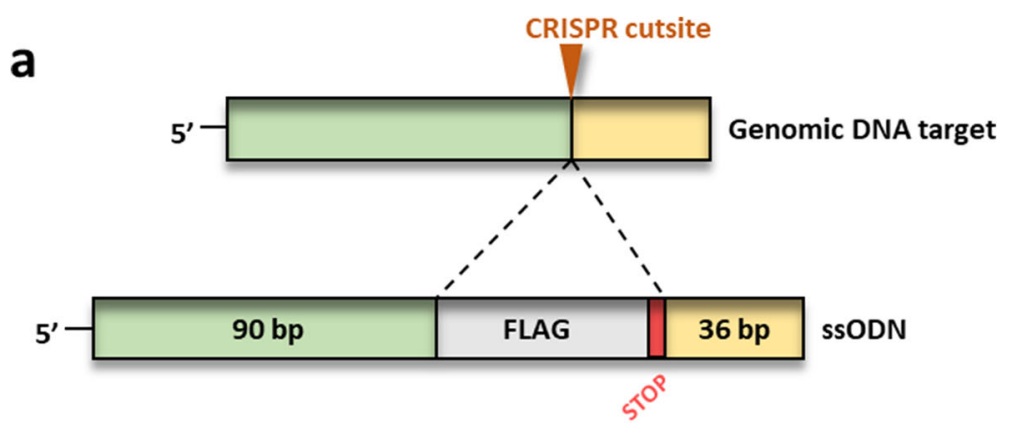

b

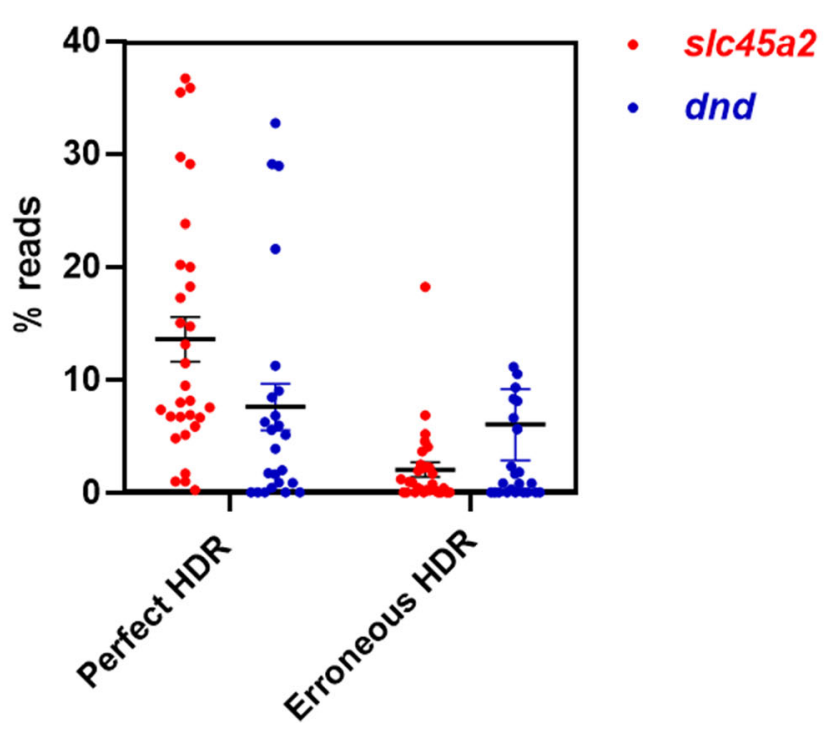

Fig. 1 s/c45a2 and dnd FLAG knock-in (1.5 $\mu \mathrm{M}$ ODN). a. Asymmetrical ODNs were designed by copying $90+36$ nucleotides on each side of the CRISPR cut site flanking the insert (indicated with a dotted line) containing the FLAG element followed by a STOP codon (TAA). b. Relative read counts per individual for $s / 445 a 2$ (red dots, $n=30$ ) and $d n d$ (blue dots, $n=24$ ). Reads with a perfect match to the entire target sequence are referred to as perfect HDR. Reads with a correct insert flanked by mismatches/indels on the $5^{\prime}$ and/or $3^{\prime}$-side are referred to as erroneous HDR. Error bars indicate SEM/group

in which perfect repair efficiency of up to $59.2 \%$ was detected. To our knowledge, this level of perfect HDR in F0 has not been reported in any other fish. Even at the lowest concentration $(0.15 \mu \mathrm{M})$, two individuals displayed 49.1 and $47.4 \%$ perfect HDR.

\section{ODN polarity affects indel locations}

In addition to reads displaying perfect HDR, we detected reads displaying erroneous repair, meaning reads with a correct FLAG-insert/SNR but also indels on the $5^{\prime}$ - and/ or 3 '-side of the insert/SNR (Figs. 1, 2 and 3b).

In our previous work using symmetrical ODNs, we revealed a strong correlation between ODN polarity and the location of these indels on either the $5^{\prime}$ - or $3^{\prime}$-side of the inserted sequence. According to this, when using a repair template with sense orientation relative to the target strand, most of the indels will end up on the $5^{\prime}$ side of the insert [27]. In the current study the asymmetrical ODNs were sense relative to the target strand (Fig.
S1a and b), and we observed that most of the indels were located on the $5^{\prime}$-side of the insert (fewer reads with perfect 5 '-reads than 3 '-reads), supporting our previous findings. A significant difference was detected for dnd KI $(P=0.034)$ and SNR $(P<0.029)$ and nonsignificant for slc45a2 KI (Fig. 4).

\section{Discussion}

This study demonstrated that asymmetrical ODNs induce efficient and precise HDR in salmon. Importantly, they appear to be more efficient than symmetrical ODNs, as compared to our previous results [27] (Fig. S2). No significant difference was detected when comparing the efficiency of FLAGKI in slc45a2 and $d n d$ (Fig. 1). This finding suggests that HDR may be applied successfully to any gene of interest.

For the first time in salmon, we performed a successful SNR by introducing a premature stop codon to the $d n d$ gene. Compared to FLAGKI we found SNR to be the 


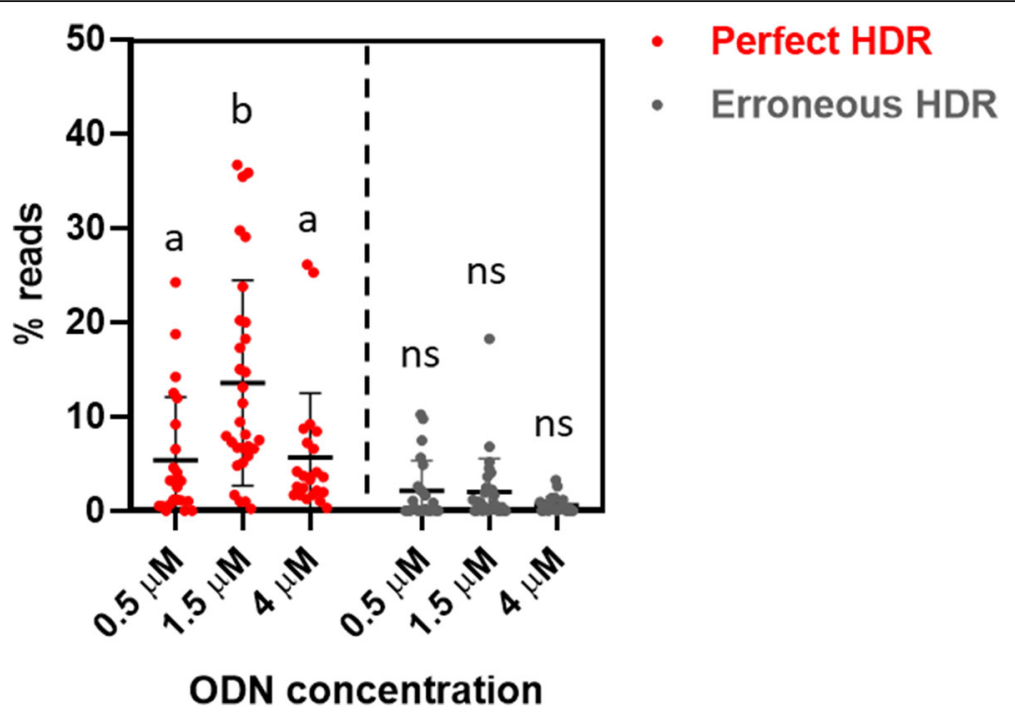

Fig. 2 slc45a2 FLAG knock-in. The asymmetrical ODN targeting s/c45a2 was tested using three different concentrations: 0.5 ( $n=23$ ), 1.5 ( $n=30$ ) and $4.0(n=23) \mu \mathrm{M}$. Sequence reads with a perfect match to the entire target sequence are referred to as perfect HDR and reads with a correct insert but mismatches/indels in the homology arms are referred to as erroneous HDR. Read counts for each sample are given in \% of the total number of reads. The error bars indicate SEM/group. Different lowercase letters indicate significant differences $(P<0.05)$, ns $=$ non-significant

most efficient approach. We speculate that the high individual HDR efficiency obtained for SNR is due to the lack of insert, as editing efficiency has been shown to be sensitive to insert size [33]. For some genes and applications, it may not be relevant to use HDR to insert DNA, but rather to obtain SNR which can translate into for example single amino acid changes, or like here, premature stop codon formation. One such example is the vgll3 gene which contains two missense SNPs, resulting in either early or late maturity in Atlantic salmon [3]. Developing precise gene editing technology to make such small edits may therefore be a useful tool in NBT, enabling introgression of natural beneficial variants into aquaculture strains. When CRISPR/Cas9 is used to make a traditional $\mathrm{KO}$ through NHEJ, one of the challenges is that the mutation can be in-frame and therefore potentially silent. SNR could solve this by insertion of a novel stop codon, and as such increase the levels of functional KO mutations by decreasing the chance of in-frame indels.

Although we found HDR efficiency to be dependent on template concentration, several parameters may contribute to this, such as fluctuation in temperature and variable presence of parasites (mostly Saprolegnia parasitica) between tanks. This often results in variable lethality, unrelated to injections as this is observed also in non-injected groups. Also, during the microinjection procedure there will be inevitable variation in the volume injected into each fertilized egg. Performing precise microinjections by hand can be challenging due to the opaque salmon eggs, and personal skills will influence the outcome. Technical aspects will also matter, such as variation in the diameter of the needle opening and the egg quality. When sampling the best phenotypic albino individuals there were visible difference in mosaicism between the groups injected with low $(0.15 / 0.5 \mu \mathrm{M})$ and high $(4 \mu \mathrm{M})$ template concentration (Fig. S3). This was true for both the slc45a2 and $d n d$ experiments. It is therefore conceivable that the mosaicism observed for the high dose group ( $4 \mu \mathrm{M} \mathrm{ODN})$ is due to toxicity of the injection mix when the injected volume is high. We hypothesize that the surviving eggs received a lower volume of the injection mix, and therefore also a lower dose of the cas 9 and guide RNA resulting in a more mosaic phenotype. Taking this into account, it could be an advantage to use the lowest possible ODN concentration to avoid unnecessary DNA induced toxicity, which would also allow editing multiple genes at the same time.

\section{Conclusion}

We show that it is possible to use CRISPR/Cas9-induced HDR in NBT to obtain desirable traits. SNR is a promising tool to insert favorable alleles in farmed salmon and, considering the long generation time, more convenient than crossing in traits through conventional breeding. Moreover, this could also be an advantage for aquaculture species in general (e.g trout, sea bass, tilapia). This technology offers an exciting opportunity to insert traits of interest into the recently demonstrated fertile but genetically sterile salmon [26]. This fish will produce sterile offspring and may therefore represent the future 
a

\section{CRISPR cutsite}

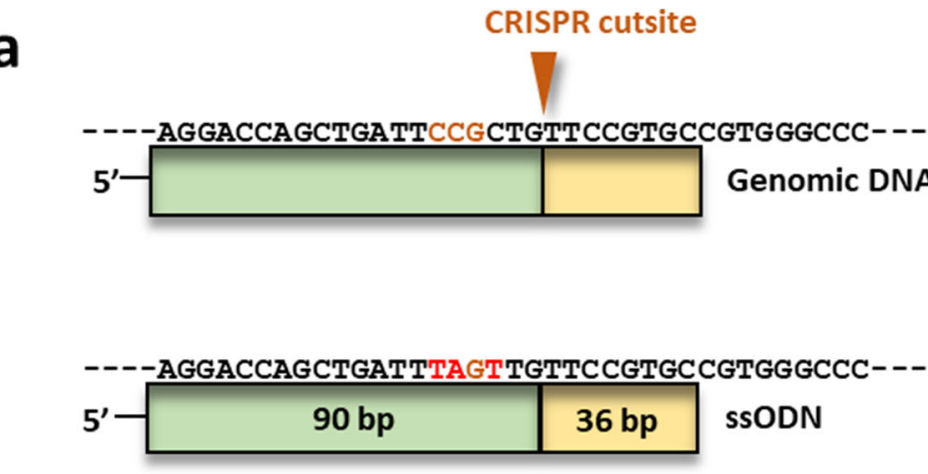

b

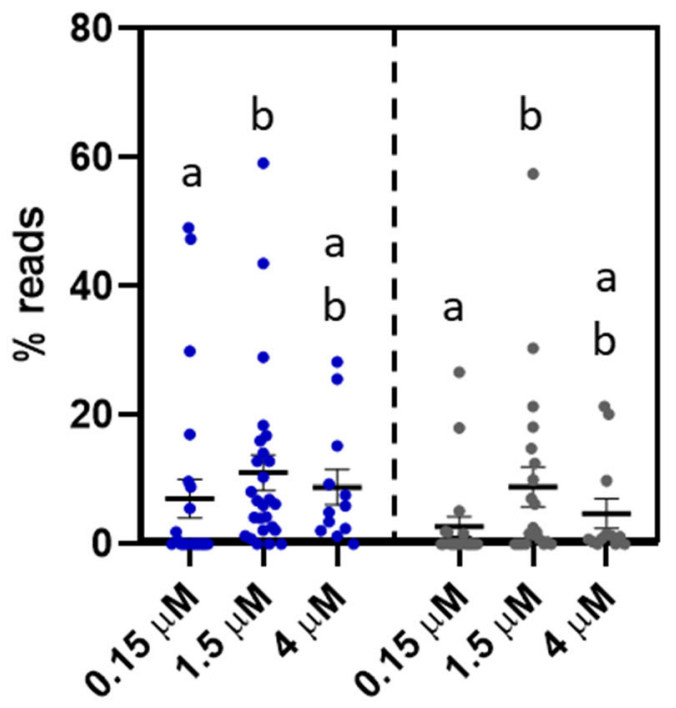

- Perfect HDR

- Erroneous HDR

\section{ODN concentration}

Fig. 3 Single nucleotide replacement in $d$ nd. a. An asymmetrical ODN targeting dnd was designed with $90+36$ nucleotides on each side of the CRISPR cut site and three nucleotides were changed. PAM site is shown with brown letters, and novel nucleotides with red letters. $\mathbf{b}$. HDR rates for three different ODN concentrations; $0.15(n=24), 1.5(n=26)$ and $4.0 \mu \mathrm{M}(n=12)$. Sequence reads with a perfect match to the entire target sequence are referred to as perfect HDR (blue) and reads with a correct SNR but mismatches/indels in the homology arms are referred to as erroneous HDR (gray). Read counts for each sample are given in \% of the total number of reads. Error bars indicate SEM/group. Different lowercase letters indicate significant differences $(P<0.05)$

salmon aquaculture by combining sterility and other favorable traits induced by HDR, such as disease resistance.

\section{Methods}

Preparation of Cas9 RNA, gRNAs and ODNs

The CRISPR target sequences for slc $45 a 2$ and $d n d$ are described in Edvardsen et al. [5], and Wargelius et al. [7], respectively. Preparation of gRNAs and cas 9 mRNA was performed as previously described [5, 27]. The RNeasy MiniKit spin column (Qiagen) was used to purify the gRNA. The ODNs were ordered from Integrated
DNA Technologies (Leuven, Belgium). The ODN design is based on Richardson et al. [30]

\section{Microinjection}

Salmon eggs and sperm were delivered by Mowi (Hauglandshella, Askøy, Norway). Fertilization and microinjections were carried out as described previously [5] using $50 \mathrm{ng} / \mu \mathrm{l}$ gRNA and $150 \mathrm{ng} / \mu \mathrm{l}$ cas 9 mRNA in nuclease free water and a FemtoJet ${ }^{\circ} 4 \mathrm{i}$ (Eppendorf) microinjector. The ODNs were added to the injection mix with a final concentration of $0.15,0.5,1.5$ or $4 \mu \mathrm{M}$. The selection of ODN concentrations were based on Straume 


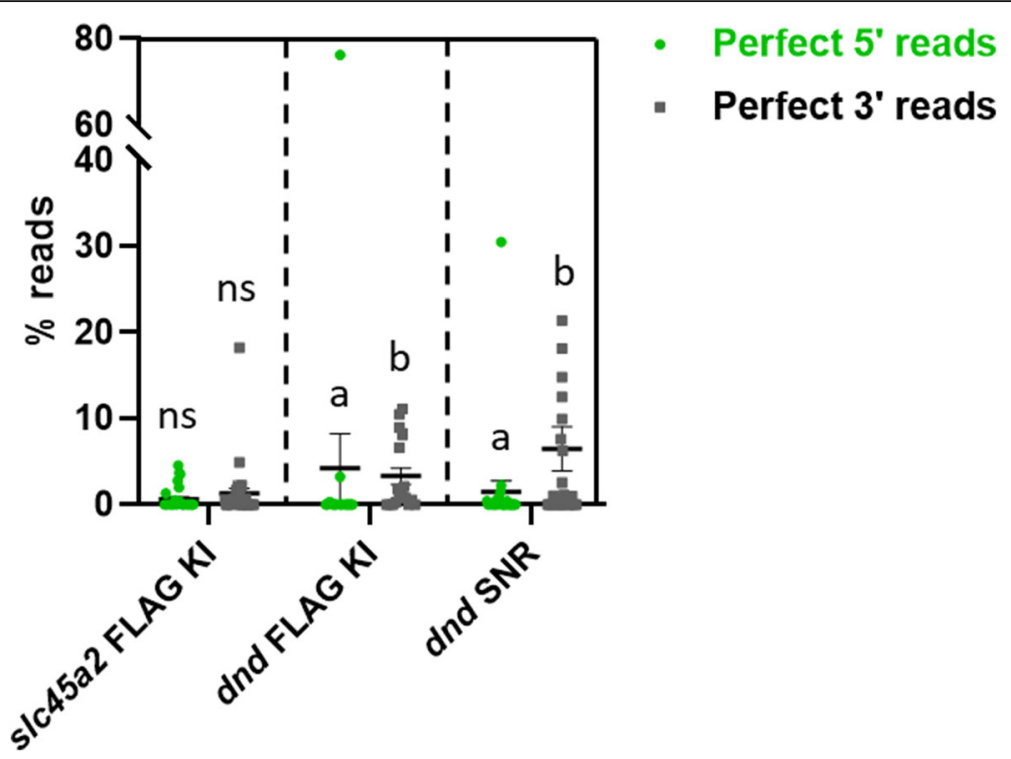

Fig. 4 Variation in indel locations. Here, we distinguished between reads with a perfect match to the $5^{\prime}$ - or $3^{\prime}$-side of the FLAG insert/SNR. The asymmetrical ODNs were compared at $1.5 \mu \mathrm{M}$. Green dots represent perfect $5^{\prime}$ reads and squares represent perfect 3' reads. Read counts for each sample are given in \% of the total number of reads. Error bars indicate SEM/group. The groups slc45a2 FLAG KI $(n=30)$, dnd FLAG KI $(n=19)$ and dnd SNR $(n=24)$ were analyzed separately. Different lowercase letters indicate significant differences $(P<0.05)$

et al. [27] and Boel et al. [28] Non-injected embryos were used as controls for all experiments.

\section{Analysis of mutants}

As described previously [27] slc45a2 mutants were selected based on visual inspection of newly hatched larvae ( $\sim 680$ day degrees). When editing $d n d$, we also added the slc45a2 gRNA to the injection mix to obtain a visual phenotype, and thus make it easier to select the mutants. DNA was extracted from caudal fins using DNeasy Blood \& Tissue kit (Qiagen). DNA extracted from the fin has previously been shown to be broadly representative for the whole fish [5,26]. A fragment covering the entire CRISPR target sites for slc45a2 and dnd was amplified with a two-step fusion PCR (as described in Gagnon et.al 2014) to prepare for Illumina MiSeq. The following primers (gene specific sequence indicated in capital letters) were used in the first PCR-step for slc45a2:

5 ' -tctttccetacacgacgetcttccgatctCAGATGTCCA-

GAGGCTGCTGCT and.

5 '-tggagttcagacgtgtgctcttccgatctTGCCACAGCCTCAGAATGTACA. The following primers (gene specific sequence indicated in capital letters) were used in the first PCR-step for $d n d$ :

5 ' -tctttccctacacgacgctcttccgatctGGGGAAAGGCTAGGGAGAGA and.

5 '-tggagttcagacgtgtgctcttccgatct CGCTGAAGTT.

\section{Analysis of MiSeq data}

Read counts were reported for variants containing the inserted or edited sequence, separating those with a perfect match to the entire target sequence (referred to as perfect HDR), and those with a correct insert sequence/ $\mathrm{SE}$, but mismatches in the rest of the target sequence (referred to as erroneous HDR). In addition, read counts were reported for wild type sequences.

The settings applied for filtering, trimming and variant calling of the MiSeq reads are illustrated in Fig. S4, and described below:

Fastq files were filtered and trimmed with the following specifications; primer sequences were used to demultiplex reads from different amplicons on the same sequencing run, minimum read length was set to $100 \mathrm{bp}$, and forward and reverse reads were assembled to correct sequencing errors (minimum overlap between forward and reverse reads was set to $150 \mathrm{bp}$ for slc45a2 and 200 bp for $d n d$, and allowing maximum $20 \%$ mismatches between forward and reverse reads in the overlap region). Assembled reads were combined with forward reads that did not pass the assembly thresholds. Variants were then called using positions 20-200 for slc45a2 and positions 60-230 for $d n d$. All bases with base quality $<20$ were converted to N's, and maximum 5 N's were allowed per read. Identical reads were then grouped (referred to as variants), and variants that only differed by up to $5 \mathrm{~N}$ 's were grouped if none of the variants differed by any nucleotides. For each group, the variant with the least N's 
was chosen as representative. We only retained variants supported by a minimum of 100 reads and variants were grouped if they differed by up to $5 \mathrm{~N}$ 's if none of the variants differed by any nucleotides.

\section{Statistical analyses}

D'Agostino Person normality test (column statistics) were used to assess normal distribution of the data. None of the groups displayed normal distribution, and we carried on with non-parametric analyses. When analyzing more than two groups, non-parametric statistical analyses were performed using a Kruskall-Wallis test, followed by Dunn's multiple comparison test. When analyzing two groups, a Mann-Whitney rank test, or a Wilcoxon paired test was performed. The tests were carried out using GraphPad Prism 8.0.1.

\section{Supplementary Information}

The online version contains supplementary material available at https://doi. org/10.1186/s12864-021-07823-8

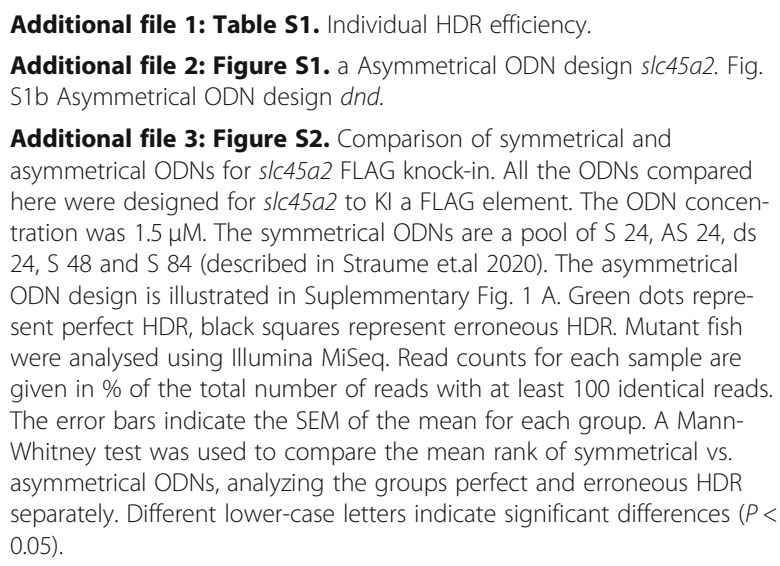

Additional file 4: Figure S3. Example of fry sampling based on visual inspection of pigmentation. Fig. S3 a s/c45a2 FLAG KI $(0.5 \mu \mathrm{M})$. Fig. S3 b slc45a2 FLAG KI $(4 \mu \mathrm{M})$. Fig. S3 c dnd SNR $(0.15 \mu \mathrm{M})$. Fig. S3 $d$ dnd SNR $(4 \mu \mathrm{M}) .{ }^{*}$ : wild type.

Additional file 5: Figure S4. Illustration of the settings applied for filtering, trimming and variant calling of the MiSeq reads. Fasta files were filtered and trimmed with the following specifications; primer sequences were used to demultiplex reads from different amplicons on the same sequencing run, minimum read length was set to $100 \mathrm{bp}$, and forward and reverse reads were assembled to correct sequencing errors (minimum overlap between forward and reverse reads was set to $150 \mathrm{bp}$ for s/c45a2 and $200 \mathrm{bp}$ for dnd and allowing at most 20\% mismatches between forward and reverse reads in the overlap region). Assembled reads were combined with forward reads that did not pass the assembly thresholds. Variants were then called using positions 20-200 for s/c45a2 and positions $60-230$ for $d$ nd. All bases with base quality $<20$ were converted to N's, and maximum $5 \mathrm{~N}$ 's were allowed per read. Identical reads were then grouped (referred to as variants) $\rightarrow$ variants that were only differing by up to $5 \mathrm{~N}$ 's were grouped if none of the variants differed by any nucleotides $\rightarrow$ for each group the variant with the least N's was chosen as representative $\rightarrow$ only retained variants supported by a minimum of 100 reads $\rightarrow$ variants were grouped if they differed by up to $5 \mathrm{~N}$ 's if none of the variants differed by any nucleotides. Finally, read counts were reported for the variants containing the inserted or edited sequence, separating those with a perfect match to the entire target sequence, and those with a correct insert sequence, but mismatches in the rest of the target sequence. In addition, read counts were reported wild type sequences.

\section{Acknowledgements}

We would like to thank Lise Dyrhovden and Ivar Helge Matre for their assistance in rearing. We are also grateful for the assistance of Hanne Sannæs and Ida Kristin Mellerud for running the MiSeq, Aquagen (Trondheim, Norway) and Mowi (Hauglandshella, Askøy, Norway) for providing eggs and sperm.

\section{Authors' contributions}

R.B.E., A.H.S., S.L. and A.W. designed the project. A.H.S., E.K.S., H.G. and R.B.E. performed the microinjections. A.H.S. and R.B.E. designed the ODN templates and collected the tissue samples. A.H.S. made the gRNA and Cas9 RNA, purified the DNA and ran the PCR screening. K.O.S. prepared the Illumina sequencing libraries. E.K.S. performed the bioinformatic analysis of the HTS data. A.H.S., A.W. and R.B.E. analyzed the results and wrote the paper. All authors read and approved the final manuscript.

\section{Funding}

Our study was funded by the EU-COFASP project: "AQUACRISPR: Optimization of the CRISPR/Cas9 knock-in technology and application in salmon and trout".

\section{Availability of data and materials}

Data generated or analyzed during this study are included in this article (and its Supplementary Information).

\section{Declarations}

\section{Ethics approval and consent to participate}

This experiment was approved by the Norwegian Animal Research Authority (NARA, permit number 14865) and the use of these experimental animals was in accordance with the Norwegian Animal Welfare Act.

The study was carried out in compliance with the ARRIVE guidelines.

\section{Consent for publication}

Not applicable.

\section{Competing interests}

The authors declare that they have no competing interests.

\section{Author details}

${ }^{1}$ Institute of Marine Research, P.O. Box 1870, Nordnes, NO-5817 Bergen, Norway. ${ }^{2}$ The Roslin Institute and R(D)SVS, University of Edinburgh, Easter Bush Campus, Midlothian EH25 9RG, UK.

Received: 23 March 2021 Accepted: 17 June 2021

Published online: 22 July 2021

\section{References}

1. Fry JP, Mailloux NA, Love DC, Milli MC, Cao L. Feed conversion efficiency in aquaculture: do we measure it correctly? Environ Res. 2018;13:024017. https://doi.org/10.1088/1748-9326/aad007

2. Gratacap RL, Wargelius A, Edvardsen RB, Houston RD. Potential of genome editing to improve aquaculture breeding and production. Trends Genet. 2019;35(9):672-84. https://doi.org/10.1016/j.tig.2019.06.006.

3. Ayllon F, et al. The vgl/3 Locus Controls Age at Maturity in Wild and Domesticated Atlantic Salmon (Salmo salar L.) Males. PLoS Genet. 2015;11: e1005628. https://doi.org/10.1371/journal.pgen.1005628.

4. Taranger GL, Karlsen $\varnothing$, Bannister RJ, Glover KA, Husa V, Karlsbakk E, et al. Risk assessment of the environmental impact of Norwegian Atlantic salmon farming. ICES J Mar Sci. 2015;72(3):997-1021. https://doi.org/10.1093/ icesjms/fsu132.

5. Edvardsen RB, Leininger S, Kleppe L, Skaftnesmo KO, Wargelius A. Targeted mutagenesis in Atlantic salmon (Salmo salar L.) using the CRISPR/Cas9 system induces complete knockout individuals in the F0 generation. PLoS One. 2014;9:e108622. https://doi.org/10.1371/journal.pone.0108622. 
6. Wargelius A. Application of genome editing in aquatic farm animals: Atlantic salmon. Transgenic Res. 2019;28(S2):101-5. https://doi.org/10.1007/ s11248-019-00163-0.

7. Wargelius A, Leininger S, Skaftnesmo KO, Kleppe L, Andersson E, Taranger $\mathrm{GL}$, et al. Dnd knockout ablates germ cells and demonstrates germ cell independent sex differentiation in Atlantic salmon. Sci Rep. 2016;6(1):21284. https://doi.org/10.1038/srep21284

8. Alex K, Datsomor NZ, Li K, Olsen RE, Jin Y, Vik JO, et al. CRISPR/Cas9mediated ablation of elovl2 in Atlantic salmon (Salmo salar L.) inhibits elongation of polyunsaturated fatty acids and induces Srebp-1 and target genes. Sci Rep. 2019;9:7533. https://doi.org/10.1038/s41598-019-43862-8.

9. Baloch AR, et al. Dnd1 Knockout in Sturgeons By CRISPR/Cas9 Generates Germ Cell Free Host for Surrogate Production. Animals (Basel). 2019;9. https://doi.org/10.3390/ani9040174

10. Chen J, Jiang D, Tan D, Fan Z, Wei Y, Li M, et al. Heterozygous mutation of eEF1A1b resulted in spermatogenesis arrest and infertility in male tilapia, Oreochromis niloticus. Sci Rep. 2017:7(1):43733. https://doi.org/10.1038/ srep43733.

11. Chen J, Wang W, Tian Z, Dong Y, Dong T, Zhu H, et al. Efficient gene transfer and gene editing in Sterlet (Acipenser ruthenus). Front Genet. 2018;9: 117. https://doi.org/10.3389/fgene.2018.00117.

12. Cleveland BM, Yamaguchi G, Radler LM, Shimizu M. Editing the duplicated insulin-like growth factor binding protein-2b gene in rainbow trout (Oncorhynchus mykiss). Sci Rep. 2018;8(1):16054. https://doi.org/10.1038/s41 598-018-34326-6.

13. Elaswad A, et al. Effects of CRISPR/Cas9 dosage on TICAM1 and RBL gene mutation rate, embryonic development, hatchability and fry survival in channel catfish. Sci Rep-Uk. 2018;8. https://doi.org/10.1038/s41598-018-3473 8-4.

14. Feng RJ, et al. Retinoic acid homeostasis through aldh1a2 and cyp26a1 mediates meiotic entry in Nile tilapia (Oreochromis niloticus). Sci Rep-Uk. 2015;5. https://doi.org/10.1038/srep10131.

15. Jiang DN, et al. CRISPR/Cas9-induced disruption of wt $1 a$ and wt 16 reveals their different roles in kidney and gonad development in Nile tilapia. Dev Biol. 2017;428(1):63-73. https://doi.org/10.1016/j.ydbio.2017.05.017.

16. Khalil K, et al. Generation of Myostatin Gene-Edited Channel Catfish (Ictalurus punctatus) via Zygote Injection of CRISPR/Cas9 System. Sci Rep-Uk. 2017:7. https://doi.org/10.1038/s41598-017-07223-7.

17. Kishimoto K, Washio Y, Yoshiura Y, Toyoda A, Ueno T, Fukuyama H, et al. Production of a breed of red sea bream Pagrus major with an increase of skeletal muscle mass and reduced body length by genome editing with CRISPR/Cas9. Aquaculture. 2018;495:415-27. https://doi.org/10.1016/j.aqua culture.2018.05.055.

18. Li M, Sun Y, Zhao J, Shi H, Zeng S, Ye K, et al. A tandem duplicate of antiMullerian hormone with a missense SNP on the $Y$ chromosome is essential for male sex determination in Nile Tilapia, Oreochromis niloticus. PLoS Genet. 2015:11(11):e1005678. https://doi.org/10.1371/journal.pgen.1005678.

19. Li M, Yang H, Zhao J, Fang L, Shi H, Li M, et al. Efficient and heritable gene targeting in tilapia by CRISPR/Cas9. Genetics. 2014;197(2):591-9. https://doi. org/10.1534/genetics.114.163667.

20. Li MH, et al. Retinoic acid triggers meiosis initiation via stra8-dependent pathway in southern catfish, Silurus meridionalis. Gen Comp Endocrinol. 2016:232:191-8. https://doi.org/10.1016/j.ygcen.2016.01.003.

21. Li, M. H., Liu, X. Y., Dai, S. F., Xiao, H. S. \& Wang, D. S. High Efficiency Targeting of Non-coding Sequences Using CRISPR/Cas9 System in Tilapia. G3-Genes Genom Genet 9, 287-295, doi:https://doi.org/10.1534/g3.118.2 00883 (2019).

22. Xie QP, He X, Sui YN, Chen LL, Sun LN, Wang DS. Haploinsufficiency of SF-1 causes female to male sex reversal in Nile Tilapia, Oreochromis niloticus. Endocrinology. 2016;157(6):2500-14. https://doi.org/10.1210/en.2015-2049.

23. Zhang XB, et al. Isolation of Doublesex- and Mab-3-Related Transcription Factor 6 and Its Involvement in Spermatogenesis in Tilapia. Biol Reprod. 2014;91. https://doi.org/10.1095/biolreprod.114.121418.

24. Zhong ZM, et al. Targeted disruption of sp7 and myostatin with CRISPR-Cas9 results in severe bone defects and more muscular cells in common carp. Sci Rep-Uk. 2016;6. https://doi.org/10.1038/srep22953.

25. SimoraRMC, et al. CRISPR/Cas9-mediated knock-in of alligator cathelicidin gene in a non-coding region of channel catfish genome. Sci Rep. 2020; 10(1):22271. https://doi.org/10.1038/s41598-020-79409-5.
26. Guralp $\mathrm{H}$, et al. Rescue of germ cells in dnd crispant embryos opens the possibility to produce inherited sterility in Atlantic salmon. Sci Rep. 2020; 10(1):18042. https://doi.org/10.1038/s41598-020-74876-2.

27. Straume AH, Kjærner-Semb E, Ove Skaftnesmo K, Güralp H, Kleppe L, Wargelius A, et al. Indel locations are determined by template polarity in highly efficient in vivo CRISPR/Cas9-mediated HDR in Atlantic salmon. Sci Rep. 2020;10(1):409. https://doi.org/10.1038/s41598-019-57295-w.

28. Boel A, et al. CRISPR/Cas9-mediated homology-directed repair by ssODNs in zebrafish induces complex mutational patterns resulting from genomic integration of repair-template fragments. Dis Model Mech. 2018;11. https:// doi.org/10.1242/dmm.035352.

29. Wierson WA, et al. Efficient targeted integration directed by short homology in zebrafish and mammalian cells. Elife. 2020;9. https://doi.org/10.7554/eLife. 53968.

30. Richardson CD, Ray GJ, DeWitt MA, Curie GL, Corn JE. Enhancing homologydirected genome editing by catalytically active and inactive CRISPR-Cas9 using asymmetric donor DNA. Nat Biotechnol. 2016;34:339. https://doi.org/1 $0.1038 / \mathrm{nbt} .3481$

31. Sanjurjo-Soriano C, Erkilic N, Baux D, Mamaeva D, Hamel CP, Meunier I, et al. Genome editing in patient iPSCs corrects the Most prevalent USH2A mutations and reveals intriguing mutant mRNA expression profiles. Mol Ther Methods Clin Dev. 2020;17:156-73. https://doi.org/10.1016/j.omtm.201 9.11.016.

32. Paix A, Schmidt H, Seydoux G. Cas9-assisted recombineering in C. elegans: genome editing using in vivo assembly of linear DNAs. Nucleic Acids Res. 2016:44:e128, https://doi.org/10.1093/nar/gkw502.

33. Paix A, Folkmann A, Goldman DH, Kulaga H, Grzelak MJ, Rasoloson D, et al. Precision genome editing using synthesis-dependent repair of Cas9induced DNA breaks. Proc Natl Acad Sci U S A. 2017;114(50):E10745-54 https://doi.org/10.1073/pnas.1711979114.

\section{Publisher's Note}

Springer Nature remains neutral with regard to jurisdictional claims in published maps and institutional affiliations.
Ready to submit your research? Choose BMC and benefit from:

- fast, convenient online submission

- thorough peer review by experienced researchers in your field

- rapid publication on acceptance

- support for research data, including large and complex data types

- gold Open Access which fosters wider collaboration and increased citations

- maximum visibility for your research: over $100 \mathrm{M}$ website views per year

At $\mathrm{BMC}$, research is always in progress.

Learn more biomedcentral.com/submissions 